\title{
Beiträge zur Kenntniss der Microlepidopteren-Fauna der Erzherzogthümer 0esterreich ob und unter der Enns und Salzburgs.
}

\author{
Von Josef Mann in Wien.
}

(2. Fortsetzung.)

Gymnancycla Z.

Canella Hb. 289. - 5, 6, in der Wiener Gegend als Falter selten, durch Zucht häufig zu erhalten. Raupe an den Stengeln der Salsola Kali, Bisamberg, Klederling.

\section{Spermatophthora Led.}

Hornigii Led. z. b. Ver. 1852.

6,7 , am Bisamberg und in der Mödlinger Gegend, auch im Prater. Raupe auf Atriplex patula in Gespinnsten.

\section{Asarta Z.}

Aethiopella Dup. - 8, auf der Raxalpe und auf der Heuplacke am Schneeberge, selten.

\section{Catastia $\mathrm{Hb}$.}

Marginea Schiff. - 6,7 , bei Rannabrunn, auf den Schneeberger Alpen, nicht selten.

var. Auriciliella Hi. 340. - \%, auf der Heuplacke, Alpeleck und Sonnenwendstein auf Wiesen, selten, auf dem Grossglockner häufig.

\section{Hypochalcia $\mathrm{Hb}$.}

Melanella Tr. $-5,6$, von Mauer bis Vöslau auf Berglehnen und Bergwiesen, nicht selten.

Ahenella $\mathbf{Z k}$. $-5,6$, überall in der Wiener Gegend, auch auf Alpen, häufig. Raupe auf Helianthemum in röhrenförmigen Gespinnsten.

Rubiginella Tr. H. S. $196 .-6$, Grinzing auf einer grasreichen Berglehne, selten.
Dignella Hb. $35 .-5,6$, Türkenschanze, Kahlenberg auf Berglehnen und trockenen Bergwiesen, selten.

Lignella Hb. 5\% - 6, \%, Grinzing, am Kahlenberg, Hermannskogl, Bisamberg, selten.

Decorella Hb. 301. - 6, im Marchfeld auf trockenen Hutweiden.

var. Germarella Zk. - 6, Eichkogl, Baden, Mödling auf Berglehnen um junges Gebüsch, sehr selten.

\section{Eucarphia $\mathrm{Hb}$.}

Vinetella $\mathbf{F}$. $-6,7$, überall um Wien auf Bergwiesen, nicht selten.

Illignella Z . - 5, Mödling, Baden etc. auf Berglehnen und Hutweiden, ziemlich selten.

\section{Epischnia $\mathrm{Hb}$.}

Prodromella Hb. 254. - 8, von Mauer bis Baden auf grasreichen Berglehnen und Wiesen, selten.

Illotella Z. Is. 1839 . - 7, auf dem Bisamberge bei der Pyramide und auf dem Eichkogl, selten.

\section{Cryptoblabes Z.}

Bistriga $\mathrm{Hw}$ - -6 , in der ehemaligen Brigittenau, im Prater an Zäunen, nicht selten. Raupe auf Almus in einem Gewebe.

\section{Brephia Hein.}

Compositella Tr. - 5, 6, 8, von Mauer bis Vöslau auf Berglehnen, Bergwiesen, nicht selten. 


\section{Acrobasis Z.}

0btusella Hb. 215. F. R. T. 57. 3. - 7, auf Pyrus communis, Mauer, Mödling, Brühl in Obstgärten, selten. Raupe lebt auf Pyrus communis und P. Malus in Gespinnsten. Auch in der Wiener Vorstadt Hundsthurm in Gärten.

Consociella $\mathbf{H b}$. 328. -6.7 , in der Wiener Gegend in jungen Gebüschen, besonders um Prunus spinosa, nicht häufig. Raupe auf Quercus, auch anf Prunus spinosa.

Tumidella Zk. -7 , in Wäldchen von Quercus in der Wiener Gegend, selten. Raupe in zusammengesponnenen Blättern von Quercus.

Rubrotibiella F. R. T. 60. 2. - 7, 8, in den Remisen bei Tivoli, Laaerberg, Mauer, Siegenfeld, nicht gar selten. Raupe auf Quercus in zusammengesponnenen Blättern.

\section{Trachonitis $\mathrm{Z}$.}

Cristella Hb. 76. H. S. 206. -6 , bei Tivoli, Mauer, Eichkogl um Gebüsche und Hecken, ziemlich selten. Raupe auf Prunus spinosa, Quercus und Evonymus in zarten Gespinnsten an Blättern und Stielen.

\section{Myelois Z.}

Rosella Sc. - 6, in der Wiener Gegend auf Berglehnen und Bergwiesen.

Cirrigerella Zk. - 6, 7, Mödling, Baden, auf grasreichen Lehnen, auch auf Hutweiden, sehr selten.

Cribrum Schiff. - 6, überall auf Hutweiden und in Gebüschen, wo viele Carduns sind, nicht selten. Raupe lebt in den Stengeln von Carduus und überwintert als solche darin.

Crudella Z. - 7, im Marchfeld: Oberweiden, dann beim Strasshof auf Hutweiden, sehr selten.

Dulcella Z. -6 , Remisen bei Tivoli, Bisamberg, Eichkogl um Hecken, sehr selten.
Legatella $\mathbf{H b} .71 .-7$, in der Wiener Gegend in Remisen und um junge Gebüsche. Raupe auf Rhammus Frangula.

Suavella Zk. - 6, 7, in der ganzen Umgebung Wiens nicht selten um Prunus spinosa. Raupe auf Prunus spinosa in röhrenförmigen Gespinnsten.

Advenella Zk. - 7, in der Wiener Gegend um Rosa- und CrataegusHecken, selten. Raupe in Gängen an den Blüthen von Rosa, Crataegus und Sorbus.

Epelydella Z - 6, 7, bei Tivoli, Mauer, Baden etc. auf Prunus spinosa, sehr selten. Raupe an Prunus spinosa.

Tetricella F. $-5,6$, in den Remisen bei Tivoli und Laaerberg, dann Mauer bis Baden, nicht gar selten.

Ceratoniae Z. - In Specereihandlungen, in Magazinen. Raupe in Hülsen von Ceratonia siliqua.

\section{Glyptoteles Z.}

Leucacrinella Z. - 5, 6, im Prater und Brigittenau an Alnus und an Zäunen, sehr selten.

\section{Eccopisa Z.}

Effractella Z Is. 1848. H. S. 188. 6 , im Prater am Schüttelzaun und an der Einfriedung des Fasangartens, sehr selten.

\section{Nyctegretis $Z$.}

Achatinella Hb. 451. - 6, 7, auf Brachfeldern, Berglehnen und Wiesen, fliegt nach Sonnenuntergang. Raupe einmal auf Matricaria Chamomilla gezogen, lebt auch auf Artemisia campestris in mit Sand bekleideten Röhren an niederen Zweigen.

\section{Ancylosis Z.}

Cinnamomella Dup. - 5, auf dem Bisamberg und Eichkogl, selten.

\section{Alispa Z.}

Angustella $\mathbf{H b} .-6$, überall um Wien. Raupe im Herbst in den Früchten von Evonymus europaeus. 


\section{Zophodia Hb.}

Convolutella $\mathbf{H b}$ - 6 , in Gärten um Ribes rubrum und Grossularia in der Wiener Gegend, selten. Raupe auf den vorgenannten Sträuchern.

\section{Euzophera Z.}

Terebrella Zk. - 6, in Nadelwäldern auf dem Gahns, auch im Mauerwald, nicht hänfig. Raupe in den Zapfen von Abies excelsa et pectinata DC.

Pinguis Hw. - 6, im Prater an Zaunplanken, Maner an Häuserwänden, selten. Raupe unter der Rinde von Fraxinus excelsior.

Bigella Z. Is. 1848. H. S. $12 \%$ 7 , in Mauer in den Kieferwäldchen beim Teiche und auf dem Liechtenstein, selten.

Biviella Z. Is. 1848 . H. S. 195 . 7. Mauer, Mödling in Kieferwäldehen, selten. Raupe von Herrn Oberförster Wachtl in den männlichen Blüthen von Pinus nigricans Host entdeckt.

Oblitella Z. 1848 , H. S. 4. 107. 194, -6 , Baden, Kalvarienberg.

Welseriella Z. - 7, bei Baden auf dem Kalvarienberge und in den Alexandrowitsch'schen Anlagen, sehr selten.

Cinerosella Z. -6 , auf dem Liechtenstein und dem Rodauner Gaisberg um Artemisia gefangen, selten.

Furcatella (F. R. i. 1.) HS. - 7, am Schneeberge auf dem Alpeleck.

\section{Homoeosoma Curt.}

Nebulea Hb. 157. - 6, überall in der Wiener Gegend anf Hutweiden, Berglehnen, nicht gar selten. Raupe in Blüthenköpfen von Carduus nutans.

Nimbelia Z. $-6,7$, überall auf Hutweiden, Berglehhen und Wiesen, nicht selten.

Binaevella Hb. $383 .-6$, in der Wiener Gegend auf Berglehnen, selten. Raupe in Stengeln und Köpfen von Carduus-Arten.
Sinuella F. -6 , Prater, Tivoli, Mödling und Baden auf pflanzenreichen Hutweiden und Berglehnen.

\section{Anerastia $\mathrm{Hb}$.}

Lotella Hb. 334. - 6, auf dem Bisamberge, selten.

\section{Ephestia Gn.}

Kühniella Zell. - 6, einzeln in Mehlkammern. Raupe lebt in Kleie und Weizenmehl. (Eingeschleppt.)

Elutella Hb. 163. - 5-8, in Wohnungen, nicht selten. Raupe in Pflanzenabfällen, todten Schmetterlingen und Herbarien, wo sie Schaden verursacht, gemein.

Semirufa Hw. - 6, im Prater an Zäunen des Fasangartens, sehr selten.

Interpunctella Hb. 310 - $5-8$, in Wohnungen. Raupe von verschiedenen Abfällen, auch von todten Schmetterlingen lebend. Nach Oberförster Wa e h l auch in aufgespeicherten Samen von Zea Mays.

\section{Galleriae.}

\section{Galleria F.}

Mellonella L. -4 und 7 , überall wo Bienenzucht getrieben wird. Raupe in Bienenstöcken, sehr schädlich.

\section{Aphomia $\mathrm{Hb}$.}

Sociella L. (Colonella L. ๆ.) 6,7 , in Häusern auf Mauern, auch um Cardus in der Wiener Gegend. Raupe in Wespennestern, Abfällen von Tuch, auch in Baumschwämmen.

\section{Melissoblaptes Z.}

Bipunctanus Curt. - In der Wiener Gegend an alten Salix-Stämmen. Raupe in Holzschwämmen und Holzmoder. Herr von Hornig erzog diese Art aus den Nestern von Pollistes gallica.

Anellus Schiff., (Sociella Hb. 24.) 7, Wiener Gegend an Pappelstämmen, Zaunplanken. Raupe auf niederen Pflanzen und in morschen Rinden in röhrenförmigen Gespinnsten. 


\section{Achroea Hb.}

Grisella F. - 7, am BaumgartnerHaus auf dem Schneeberge und bei Bienenzüchtern. Raupe in den Waben der Bienenstöcke.

\section{Tortricina.}

\section{Rhacodia $\mathrm{Hb}$.}

Caudana F. - 7, im Prater auf Salix, selten. Raupe anf Salix caprea.

var. Emargana F. - 7, bei Gumpoldskirchen an Salix-Stämmen, sehr selten.

Effractana Fröl. - 8, einmal bei Mauer im Walde an Quercus-Stämmen gefangen, sehr selten.

\section{Teras Tr.}

Cristana F., nebst Varietäten. 9,10 , in den Remisen bei Tivoli, Mauer, Dornbach, Weidling-Bach. Raupe auf Quercus, Salix und Betula.

Umbrana Hb. 59. - 10, in Mauer im Gemeindewald auf Abies pectinata DC., selten. Raupe auf Salix und Quercus.

Hastiana L., und Varietäten. - 9, in den Remisen bei Tivoli, Laaerberg, Prater, Mauer etc. in jungen Gehölzen.

Maccana Tr. - 9, Mauer bei der Schiessstätte auf Calluna, sehr selten.

Mixtana Hb. 215, - 8, Mauer bei der Schiessstätte auf Erica, selten. Raupe auf Erica.

Logiana Schiff. -9 , in den Remisen bei Tivoli, auf dem Eichkogl, selten. Raupe auf Viburnum Opulus L,

Permutatana Dup. H. S. 28. - 8, in der Umgebung von Wien bei Prunus- und Crataegus-Hecken, selten. Raupe auf Crataegus Oxyacantha L., Prunus spinosa L. und Rosa.

Variegana Schiff. -6 und 8 , in der Wiener Gegend um Crataegusund Prunus-Hecken.

ab. Asperana F. Hb. 240. - 8, in Gebüschen auf der Himmelswiese bei Kalksburg, Baden, sehr selten. Raupe auf Prunus spinosa L. und Crataegus Oxyacantha L.

Boscana F. - 7, 8-11, im Prater an Ulmus-Stämmen und Holzzännen, nicht selten.

Parisiana Gn. - 8-11, im Prater an Uimus-Stämmen und Zaunplanken, nicht selten. Raupe auf Ulmus. Verpuppung in Rindenritzen.

Literana L. (Hb. 89, 90.) - 4, 7, 8, im Mauererwalde an Quercus-Stämmen, auch in Dornbach etc., selten. Raupe auf Quercus.

var. Suavana H. S. $-8,9$, in Mazer im Gemeindewald auf Quercus, sehr selten. Raupe auf Quercus.

var. Squamana F. (H. S. 2, 3, IV, p. 152, Squamulana Hb. 92-4.)

var. Irrorana Hb. 97. - 8, 9, auf Quercus im Gemeindewald von Mauer, in Dornbach, am Bisamberg etc., gemein. Raupe auf Quercus und Carpinus.

Niveana F. -9 , Weidlingbach in Birkenwaldungen. Raupe auf Betula alba.

Roscidana Hb. 103. - 7, hinter Neuwaldegg in jungem Eichengebüsch, anf dem Bisamberg, selten. Raupe auf Laubhölzern.

Lipsiana Schiff. - 5 und 8 , in den Remisen bei Tivoli, bei Mauer im Gemeindewald im Laubgebüsch.

Sponsana F. - 8, 9, in der Wiener Gegend in jungen Laubgebüschen. Raupe auf Laubhölzern, auch auf Pyrus Malus L.

Rufana Schiff. - 9, 10, in Remisen bei Tivoli, Laaerberg, Schönbrunn, Mauer etc. in jungem Gebüsch. Raupe auf Laubbäumen und Sträuchern.

Schalleriana L. -7 , bei Lainz an einem Gartenzaun einmal gefunden. Die Raupe fand ičh in Reichstadt auf Pyrus Malus L.

Comparana Hb. 284. - 8, im Prater und Weidlingbach in jungem Betulaund Populus-Gebüsch, selten.

(Fortsetzung folgt.) 


\section{$2 \mathrm{BHL}$ Biodiversity Heritage Library}

Mann, Josef Johann. 1884. "Beiträge zur Kenntniss der

Microlepidopteren-Fauna der Erzherzogthümer Oesterreich ob und unter der Enns und Salzburgs. (Anm.: 2. Fortsetzung)." Wiener entomologische Zeitung 3, 225-228. https://doi.org/10.5962/bhl.part.13850.

View This Item Online: https://www.biodiversitylibrary.org/item/43792

DOI: https://doi.org/10.5962/bhl.part.13850

Permalink: https://www.biodiversitylibrary.org/partpdf/13850

\section{Holding Institution}

Smithsonian Libraries

\section{Sponsored by}

Smithsonian

\section{Copyright \& Reuse}

Copyright Status: NOT_IN_COPYRIGHT

This document was created from content at the Biodiversity Heritage Library, the world's largest open access digital library for biodiversity literature and archives. Visit BHL at https://www.biodiversitylibrary.org. 